\title{
Methoprene modifies adipokinetic hormone characteristics in the firebug Pyrrhocoris apterus (Heteroptera: Pyrrhocoridae)
}

\author{
Dalibor KODRÍK ${ }^{1,2}$, Glenda ALQUICER ${ }^{1,2}$ and RADOMír SOCHA ${ }^{1}$ \\ ${ }^{1}$ Institute of Entomology, Biology Centre, Academy of Sciences, and ${ }^{2}$ Faculty of Science, University of South Bohemia, \\ Branišovská 31, 37005 České Budějovice, Czech Republic; e-mail: kodrik@entu.cas.cz
}

Key words. AKH, juvenile hormone analogue, lipid mobilization, lipid metabolism, ELISA

\begin{abstract}
In the present study the hypothesis that there is a feedback between juvenile hormone and adipokinetic hormones (AKHs) was investigated by topical application of the juvenoid methoprene on 9-day-old adult males of the firebug Pyrrhocoris apterus. This juvenoid $(2 \mu \mathrm{g})$ induced a significant reduction of haemolymph lipids $24 \mathrm{~h}$ after treatment; however, it did not significantly reduce the ability of Pyrap-AKH (10 pmol/bug) to mobilize fat body lipids 6-72 h after the methoprene application. The same methoprene treatment elicited a significant increase of AKH content in the CNS (central nervous system: brain + corpora cardiaca + corpora allata) of experimental males 24 and $48 \mathrm{~h}$ after the juvenoid application. A significant decrease in the AKH level in the haemolymph was recorded at the same times and under the same experimental conditions. Similar results were observed when production of the AKHs from the CNS of topically treated males was measured under in vitro conditions. It is suggested that methoprene may reduce $\mathrm{AKH}$ release from the CNS resulting in an increase in the AKH content of the CNS due to accumulation rather than stimulation of AKH synthesis. Possible consequences of this phenomenon on the physiology of P. apterus are discussed.
\end{abstract}

\section{INTRODUCTION}

The adipokinetic hormones (AKHs) in insects are synthesised, stored and released by neurosecretory cells from the corpora cardiaca (CC), a neuroendocrine gland connected to the brain. These hormones are grouped together with a chromatophorotropin, a red pigment concentrating hormone (Fernlund \& Josefsson, 1972), in the AKH/RPCH peptide family. The most important function of AKHs is the control of energy metabolism and mobilization of different kinds of energy reserves such as lipids, carbohydrates and/or certain amino acids (Gäde et al., 1997), but generally they act as typical stress hormones stimulating catabolic reactions and inhibiting synthetic reactions to combat stress and to suppress processes that are momentarily less important. Such processes, if allowed to continue, could potentially even draw on the mobilized energy, e.g., synthesis of storage nutrients (Kodrík, 2008). More than 40 insect AKHs have been isolated and characterized from representatives of many insect orders (Gäde et al., 1997; Gäde \& Goldsworthy, 2003). In Heteroptera, AKHs of 27 species of bugs (see Kodrík et al., 2010), including the firebug Pyrrhocoris apterus have been isolated and identified. Two different AKHs (Pyrap-AKH and Peram-CAH-II) were found to occur in this heteropteran species (Kodrík et al., 2000, 2002a).

$P$. apterus shows conspicuous wing polymorphism and produces both macropterous (long-winged) and brachypterous (short winged) morphs (Honěk, 1976; Socha, 1993). The macropterous adults differ from the brachypterous ones in several biochemical, physiological and behavioural parameters; e.g., a longer pre-reproductive and pre-oviposition period (Honěk, 1985; Socha \& Šula,
1996; Socha, 2006), lower level of glycogen and lipids in the fat body (Socha et al., 1997, 1998), decreased food intake (Socha et al., 1997, 1998; Socha \& Zemek, 2007) and enhanced walking and dispersal (Socha \& Zemek, 2000, 2003) associated with lower and postponed mating activity (Socha, 2004, 2008). The greater walking and dispersal activity of 10-day-old macropterous adults of $P$. apterus is positively correlated with a more intensive mobilization of lipids by AKH (Maxová et al., 2001) and higher levels of AKH in the CNS (Kodrík et al., 2003) and haemolymph (Kodrík et al., 2002b). The higher levels of AKH in 5-14-day-old spontaneously fasting adult macropters are associated with flight muscle development (Socha \& Šula, 2006), inhibition of ovarian growth in females (Socha, 2007) and delayed maturation of the accessory glands in males (Socha, 2006). The growth of the reproductive organs in both brachypterous and macropterous adults of this bug is dependent on juvenile hormone $(\mathrm{JH})$. This has been demonstrated by measuring the volume and activity of the corpus allatum (CA), the source of this hormone (Hodková \& Socha, 2006; Socha \& Hodková, 2006), allatectomy and by treating adults with an active juvenile hormone analogue (JHa) methoprene (Socha et al., 1991, 2005; Socha, 2007). This indicates that the $\mathrm{JH}$ titre in 1-14-day-old macropterous adults is probably below the threshold needed for stimulating egg production in females and growth of accessory glands in males. It appears therefore, that an increase in the titre of $\mathrm{AKH}$ in 5-14-day-old macropterous bugs is associated with the absence or very low titre of $\mathrm{JH}$ in the body. On the other hand, a decrease of the AKH titre in the CNS and haemolymph in macropterous adults older than 14 days is associated with resumption of food consumption (Socha \& Zemek, 2007), followed by full reac- 
tivation of the CA (Hodková \& Socha, 2006) and subsequent flight muscles histolysis induced by enhanced titre of JH in the body (Socha \& Šula, 2006, 2008).

It is presumed that dispersal of 5-14-day-old macropterous adults is associated with the absence or a very low titre of $\mathrm{JH}$ and high titre of $\mathrm{AKH}$ in the body, while the transition from dispersal to reproduction in adults older than 14 days is coupled with the change in the levels of these hormones in favour of JH (Socha et al., 2005). This is supported by preliminary experiments that indicate the juvenoid methoprene partially inhibits some adipokinetic characteristics in adult brachypterous females of the bugs P. apterus and Dysdercus cingulatus (Bártů et al., 2006). In addition, it was reported that $\mathrm{AKH}$ interferes with the production of eggs, because its application leads to a significant reduction in the size of the ovaries due to the inhibition of the synthesis of vitellogenins in the fat body of the locust Locusta migratoria (Carlisle \& Loughton, 1986; Moshitzky \& Applebaum, 1990) and the cricket Gryllus bimaculatus (Lorenz, 2003).

The aim of the present study was to determine the interaction between $\mathrm{JH}$ and $\mathrm{AKH}$ and confirm experimentally the possible regulatory effect of $\mathrm{JH}$ on the synthesis and/or release of $\mathrm{AKH}$ into haemolymph. Because the authentic $\mathrm{JH}$ of $P$. apterus has not been identified, the $\mathrm{JH}$ analogue methoprene, which exhibits strong JH effects on this species, was used in this study. To avoid a possible feedback effect of methoprene-stimulated synthesis of vitellogenin, ovarian growth and egg production, the work was performed on P. apterus adult males.

\section{MATERIAL AND MEHODS}

\section{Experimental animals}

A stock culture of the firebug, Pyrrhocoris apterus (L.) (Heteroptera, Insecta), established from wild populations collected at České Budějovice (Czech Republic, $\left.49^{\circ} \mathrm{N}\right)$, was used in the present study. Larvae and adults were kept in 0.51 glass jars in a mass culture (approximately 40 specimens per jar) and reared at constant temperature of $26 \pm 1{ }^{\circ} \mathrm{C}$ under long-day conditions (18L : 6D). They were supplied with linden seeds and water ad libitum, which were replenished twice a week. Freshly ecdysed adults were transferred to small 0.251 glass jars (females and males separately) and kept under the same photoperiodic, food and temperature regimes in which they developed. To avoid a putative feedback effect of ovarian growth on AKH characteristics after methoprene treatment only males were used for the experiments.

\section{Hormonal treatment}

The native $\mathrm{JH}$ of $P$. apterus has not been identified in spite of a prolonged and intensive effort (M. Hodková, pers. comm.); thus, a JHa-methoprene, was used to mimic JH action. Methoprene exhibits very high $\mathrm{JH}$ activity in various Heteroptera, including $P$. apterus. In this species it induces moulting to superlarvae, terminates reproductive diapause, induces the synthesis of vitellogenin in the fat body, 53-kDa glycoprotein in accessory glands and triggers the histolysis of indirect flight muscles in macropterous adults of both sexes (Sláma et al., 1974; Socha et al., 1991, 2004; Socha \& Šula, 2006). The methoprene used in the present study was prepared in the Research Institute of Organic Syntheses (Rybitví, Czech Republic). The 9-day-old adult experimental males were treated with 2 $\mu \mathrm{l}$ of an acetone solution of methoprene $(1 \mu \mathrm{g} / \mu \mathrm{l})$, applied topically to the abdomen under the wings according to the method previously described (Socha et al., 2005). The effects (see below) were evaluated 6, 24, 48 and $72 \mathrm{~h}$ later. Controls were treated only with acetone solution.

\section{Lipid mobilization assay}

In certain experiments, the effect of methoprene on the lipid mobilization ability of Pyrap-AKH was evaluated. Pyrap-AKH, custom-synthesized by Polypeptide Laboratories s.r.o. (Praha, Czech Republic), was dissolved in $20 \%$ methanol in Ringer saline. The experimental bug males were injected with the hormonal solution (dose $10 \mathrm{pmol}$ in $2 \mu \mathrm{l}$ of the solution - for reasons see Kodrík et al., 2000) with a syringe (10 $\mu$ l Hamilton Co., Reno, Nevada) through the metathoracic-abdominal intersegmental membrane into the thorax 6-72 h (see above) after the methoprene treatment. Controls were injected only with the solvent. The lipid content of the haemolymph samples was determined as described previously (Kodrík et al., 2000) using an assay based on the sulpho-phosphovanilin test (Zöllner \& Kirsch, 1962). Haemolymph samples were taken from the cut end of an antenna and a drop of haemolymph was leaked onto a piece of parafilm $\mathrm{M}$ and $0.5 \mu \mathrm{l}$ taken up in a micropipette (Eppendorf Varipipette 4810). The samples were collected just before and $90 \mathrm{~min}$ after the hormonal injection and used for the determination of lipids. The optical densities at $546 \mathrm{~nm}$, measured in a spectrophotometer (UV 1601 Shimadzu), were converted to $\mathrm{mg}$ lipids per $\mathrm{ml}$ haemolymph with the aid of a calibration graph based on known amounts of oleic acid.

\section{Extraction of AKH from CNS and haemolymph}

Methanolic extracts $(80 \%$ methanol) of the brains with $\mathrm{CC}$ and CA attached (CNS) were used in a competitive ELISA (see below) to determine the AKH content of the CNS of bugs 6-72 $\mathrm{h}$ (see above) after the methoprene treatment. For the ELISA determination of the endogenous AKH titre in the haemolymph, some pre-purification was essential. Haemolymph samples were extracted in $80 \%$ methanol and after centrifugation the supernatants were evaporated to dryness. Solid phase extraction (Sep Pak C18, Waters) and HPLC purification (Chromolith Performance RP-18e column, Merck) were employed before the ELISA (for details see Goldsworthy et al., 2002).

The efficiency of recovering haemolymph AKH by this extraction procedure was checked by adding 500 fmol of Pyrap-AKH to $20 \mu \mathrm{l}$ samples of haemolymph before extraction. The recovery of AKH $(74.8 \pm 8.2 \%$; mean \pm SE) was checked using ELISA (see below) and estimated from five separate parallel measurements; all respective data were corrected for this loss $(25 \%)$.

\section{In vitro assay}

After 6-72 h of methoprene or acetone treatment (see above), the CNSs of bugs were dissected, washed in Ringer saline and immediately transferred to glass tubes filled with $200 \mu \mathrm{l}$ of Grace's medium. Each tube contained the CNS of three individuals that simultaneously received the same treatment. The incubation lasted $6 \mathrm{~h}$ at room temperature. After incubation, both the medium and tissue were separately stored in plastic tubes at $-20^{\circ} \mathrm{C}$.

Prior to $\mathrm{AKH}$ determination, all samples were subjected to extraction in $80 \%$ methanol: the CNS tissue samples were sonicated in methanol, centrifuged and the supernatants evaporated like the samples from the in vivo experiment, while the samples of the medium (each $200 \mu$ l) were repeatedly mixed with methanol, centrifuged and evaporated. All these samples were kept at $-20^{\circ} \mathrm{C}$ until determination of $\mathrm{AKH}$ content by competitive ELISA. 
TABLE 1. Effect of methoprene $(2 \mu \mathrm{g})$ on the level of lipids in the haemolymph of 9-day-old P. apterus males recorded at different times after the application of methoprene; control males were only treated with acetone. Statistically significant difference at the $5 \%$ level (experimental vs. control) is indicated by asterisks. The numbers in brackets represent number of parallel repetitions $(n)$.

\begin{tabular}{ccc}
\hline $\begin{array}{c}\text { Time after treatment } \\
\text { (hours) }\end{array}$ & $\begin{array}{c}\text { Methoprene treatment: haemolymph lipid level } \\
\text { mg/ml (mean } \pm \text { S.D.) }\end{array}$ & $\begin{array}{c}\text { Acetone treatment (control): haemolymph lipid level } \\
\mathrm{mg} / \mathrm{ml}(\mathrm{mean} \pm \text { S.D.) }\end{array}$ \\
\hline 0 & & $8.63 \pm 1.33(7)$ \\
6 & $8.71 \pm 1.49(20)$ & $9.36 \pm 2.02(19)$ \\
24 & $6.86 \pm 1.03^{*}(19)$ & $9.00 \pm 3.26(19)$ \\
48 & $7.19 \pm 2.43(17)$ & $9.00 \pm 4.57(18)$ \\
72 & $7.79 \pm 1.89(19)$ & $8.08 \pm 1.40(18)$ \\
\hline
\end{tabular}

\section{Quantification of AKH by ELISA}

A competitive ELISA was used to determine the AKH content of $P$. apterus CNS (1/4 CNS equiv.), haemolymph $(20 \mu 1$ equiv.) and incubation medium $(200 \mu \mathrm{l})$ according to the protocol described earlier (Goldsworthy et al., 2002; Kodrík et al., 2003). Commercially prepared rabbit antibodies which were produced against Cys'-Pyrap-AKH (Sigma Genosys Biotechnologies Ltd.) bind with both Pyrap-AKH and Peram-CAH-II. Prior to the ELISA the 96-well microtitre plates (high binding Costar, Corning Incorporated, Corning, New York) were precoated overnight with IgG dilution $1: 10,000$ for CNS and 1 : 2000 for haemolymph and medium. A biotinylated probe prepared from Cys ${ }^{1}$-Pyrap-AKH using Biotin Long Arm Maleimide (BLAM, Vector Laboratories, UK) was used in this assay (for details see Goldsworthy et al., 2002). The Pyrap-AKH (Polypeptide Laboratories, Praha) was used as a standard for AKH quantification.

\section{Statistical analysis and presentation of the results}

The results obtained were plotted using the graphic program Prism (GraphPad Software, version 5.0, San Diego, CA, USA). The values on the graphs and in Table 1 are means of the measurements $\pm \operatorname{SD}(n=6-10$ for Fig. $1, n=6-9$ for Figs 2 and 3). Significances of the results were evaluated using Student's t-tests (at 5\% significance level). For the complex analysis of the results multivariate principal component analysis was employed using Canoco for Windows v. 4.2 software (Fig. 4) and the data were evaluated at the $5 \%$ significance level.

\section{RESULTS}

\section{Lipid mobilization}

The experiments were initiated by topical application of methoprene $(2 \mu \mathrm{g})$ on 9-day-old males. At 6, 24, 48 and $72 \mathrm{~h}$ after the JHa treatment, 10 pmol Pyrap-AKH was injected and the adipokinetic activity measured within 90 min. The results of lipid mobilization bioassay (Fig. 1A) showed that methoprene did not significantly affect the ability of the Pyrap-AKH to mobilize fat body lipids into the haemolymph. Additionally, the adipokinetic response at time $0(7.04 \pm 2.17 \mathrm{mg} / \mathrm{ml}, n=6-$ no methoprene or acetone treatment, injection of 10 pmol Pyrap-AKH) did not significantly differ from that recored for each of the stated time periods. Thus, methoprene did not substantially affect the ability of Pyrap-AKH to mobilize fat body lipids. To exclude the possibility that methoprene alone can mobilize lipids a positive control (Fig. 1B) was included. In this case, the males treated with methoprene (or acetone for controls) were injected 6, 24, 48 and $72 \mathrm{~h}$ later with $20 \%$ methanol in Ringer saline - and the intensity of lipid mobilization measured. Again, no significant changes in the haemolymph lipid level were recorded, and the values did not significantly differ from the data at time $0(0.00 \pm 1.28 \mathrm{mg} / \mathrm{ml}, n=7$ - intact males - no methoprene or acetone treatment, injection of $2 \mu 1$ Ringer saline).

A limited direct effect of methoprene on lipid metabolism was recorded for treated bugs 6-72 h after application of methoprene (Table 1). There was a decline in the

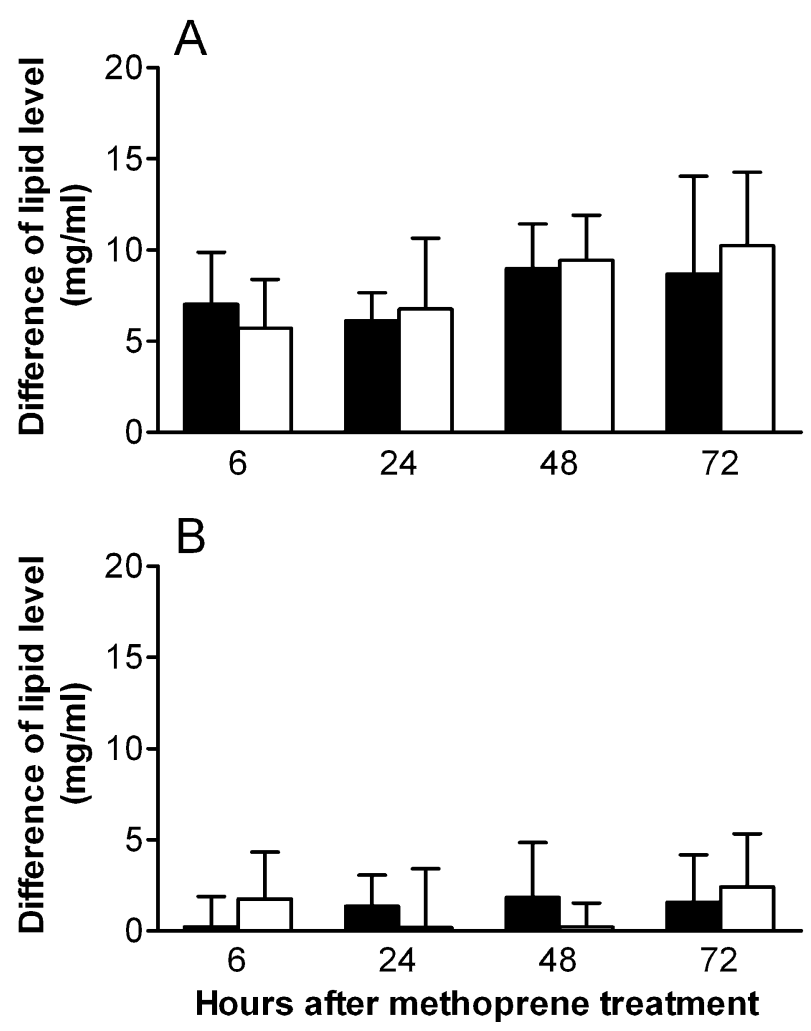

Fig. 1. (A) Effect of methoprene $(2 \mu \mathrm{g})$ on the level of lipid in haemolymph of 9-day-old $P$. apterus males injected with 10 pmol Pyrap-AKH and evaluated at 6, 24, 48 and $72 \mathrm{~h}$ after the methoprene application - solid columns; control males treated only with acetone and injected with the same dose of Pyrap-AKH - empty columns. (B) Positive control excluding any effect of the methoprene treatment on the changes in the lipid levels: the males treated with methoprene were injected with $20 \%$ methanol in Ringer saline at 6, 24, 48 and $72 \mathrm{~h}$ after the treatment - solid columns; control males were treated with acetone only and injected with $20 \%$ methanol in Ringer saline empty columns. The results are expressed as mean \pm S.D. There is no statistical difference at the $5 \%$ level between the experimental and control in either 1A or 1B. 

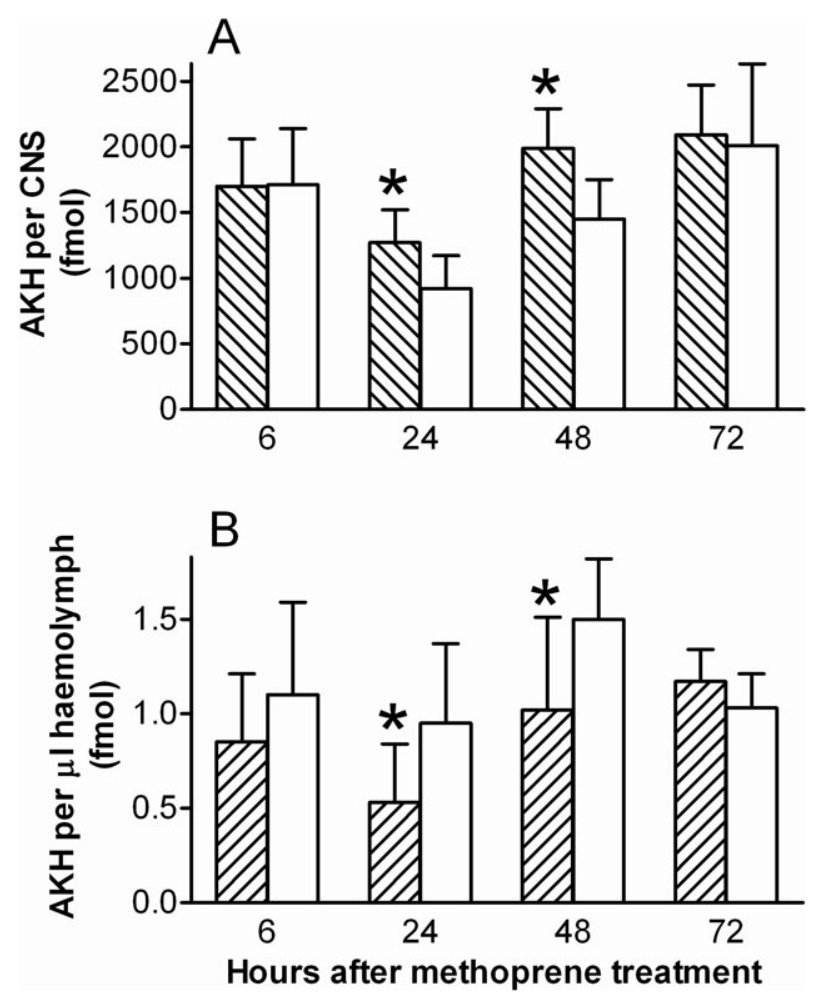

Fig. 2. Effect of methoprene $(2 \mu \mathrm{g})$ on the level of AKHs in (A) CNS (crosshatched columns) and (B) haemolymph (crosshatched columns) of 9-day-old $P$. apterus males evaluated at 6 , 24, 48 and $72 \mathrm{~h}$ after methoprene application. Control males were only treated with acetone (open columns). The results are expressed as mean \pm S.D., statistically significant differences at the $5 \%$ level (experimental vs. control) are indicated by asterisks.

lipid level, which was significant only at $24 \mathrm{~h}$ after the treatment and not at any other time.

\section{Level of AKHs in the body of firebugs}

In another series of experiments the putative effects of methoprene on the synthesis and release of AKHs from and present in the CNS of P. apterus were tested. The results showed (Fig. 2A) that 24 and $48 \mathrm{~h}$ after methoprene treatment the AKH content in the CNS of experimental males increased significantly compared with corresponding controls. The increase was not dramatic and at both time intervals was about 1.4 times that recorded for the acetone treated controls. A time interval of $6 \mathrm{~h}$ was probably too short and that of $72 \mathrm{~h}$ too long for recording significant methoprene effect. The AKH content of the CNS at time 0 (intact males, $1650 \pm 470 \mathrm{fmol}$ AKHs/bug, $n=6$ ) did not statistically differ from that of the acetone-treated control $6 \mathrm{~h}$ after treatment.

The completely opposite situation was recorded in the haemolymph of methoprene-treated firebugs (Fig. 2B). There was a significant decrease in the AKH titre 24 (1.8 fold) and 48 (1.5 fold) $\mathrm{h}$ after the treatment compared with corresponding controls. Again no significant changes were recorded at 6 and $72 \mathrm{~h}$ after the treatment. The AKH level in haemolymph of experimental bugs at time 0 (intact males, $1.10 \pm 0.04 \mathrm{fmol} \mathrm{AKHs} / \mu 1$ haemo-
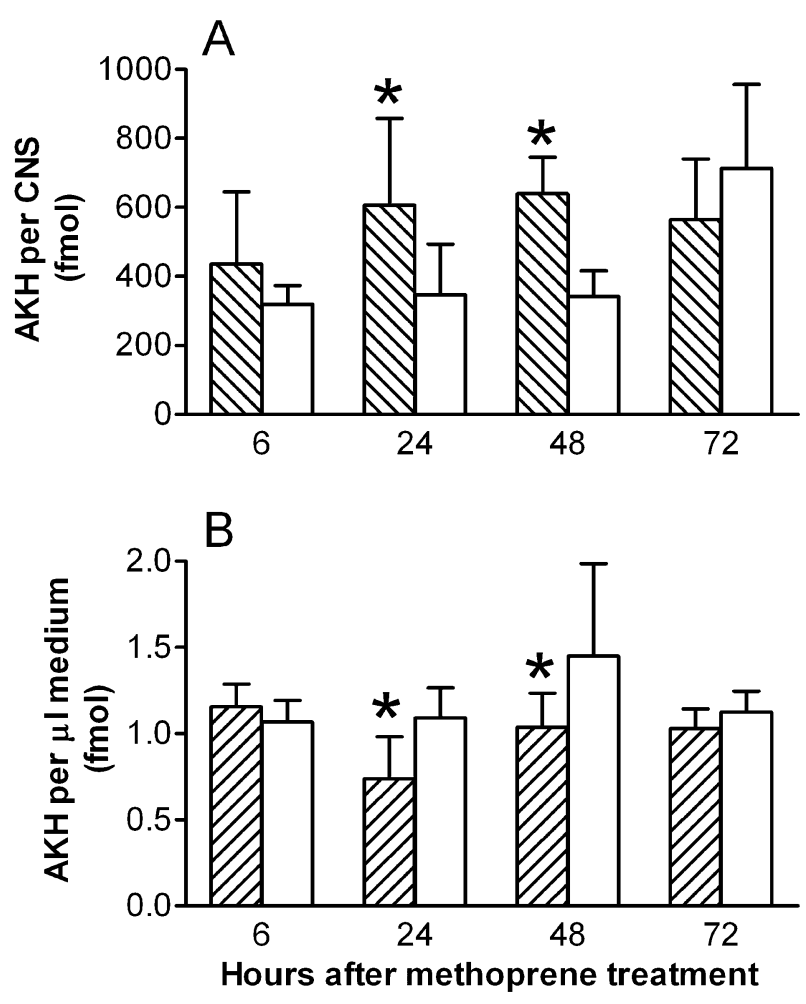

Fig. 3. Effect of methoprene $(2 \mu \mathrm{g})$ on the level of AKHs in (A) CNS of 9-day-old P. apterus males incubated in vitro for period of 6-h in Graces medium (crosshatched columns) and (B) the incubation medium (crosshatched columns). The dissection of the CNS for the incubation assay was done at different times after methoprene application (for details see Materials and Methods). Control males were only treated with acetone (open columns). The results are expressed as mean \pm S.D., statistically significant differences at the $5 \%$ level (experimental vs. control) are indicated by asterisks.

lymph, $n=4$ ) did not statistically differ from that in acetone-treated controls $6 \mathrm{~h}$ after treatment. To summarize the results presented in Figs $2 \mathrm{~A}$ and $2 \mathrm{~B}$, there is a mutual interaction between methoprene and AKHs in firebug males. After methoprene treatment the release of $\mathrm{AKH}$ from the CNS into the haemolymph ceased, and simultaneously, 24 and $48 \mathrm{~h}$ after treatment, there was a significant increase in the hormone content of the CNS. Thus, methoprene may restrict the release of $\mathrm{AKH}$ and the increase in the level of AKH content in the CNS may be the consequence of its greater accumulation, rather than stimulation, of its synthesis.

To exclude possible effects of other organs, nerve connections or humoral factors, the spontaneous production of AKHs by the CNS of $P$. apterus incubated in a medium was measured under in vitro conditions over a period of $6 \mathrm{~h}$. The content of AKHs in the CNS at the end of this incubation period was also determined. This experiment, comprising the methoprene treatment under in vivo and time conditions as mentioned above, confirmed the results obtained in vivo. The significant increase of $\mathrm{AKH}$ content in the CNS (Fig. 3A) was recorded 24 (1.7 fold) and 48 (1.9 fold) $\mathrm{h}$ after methoprene treatment, while a significant reciprocal 

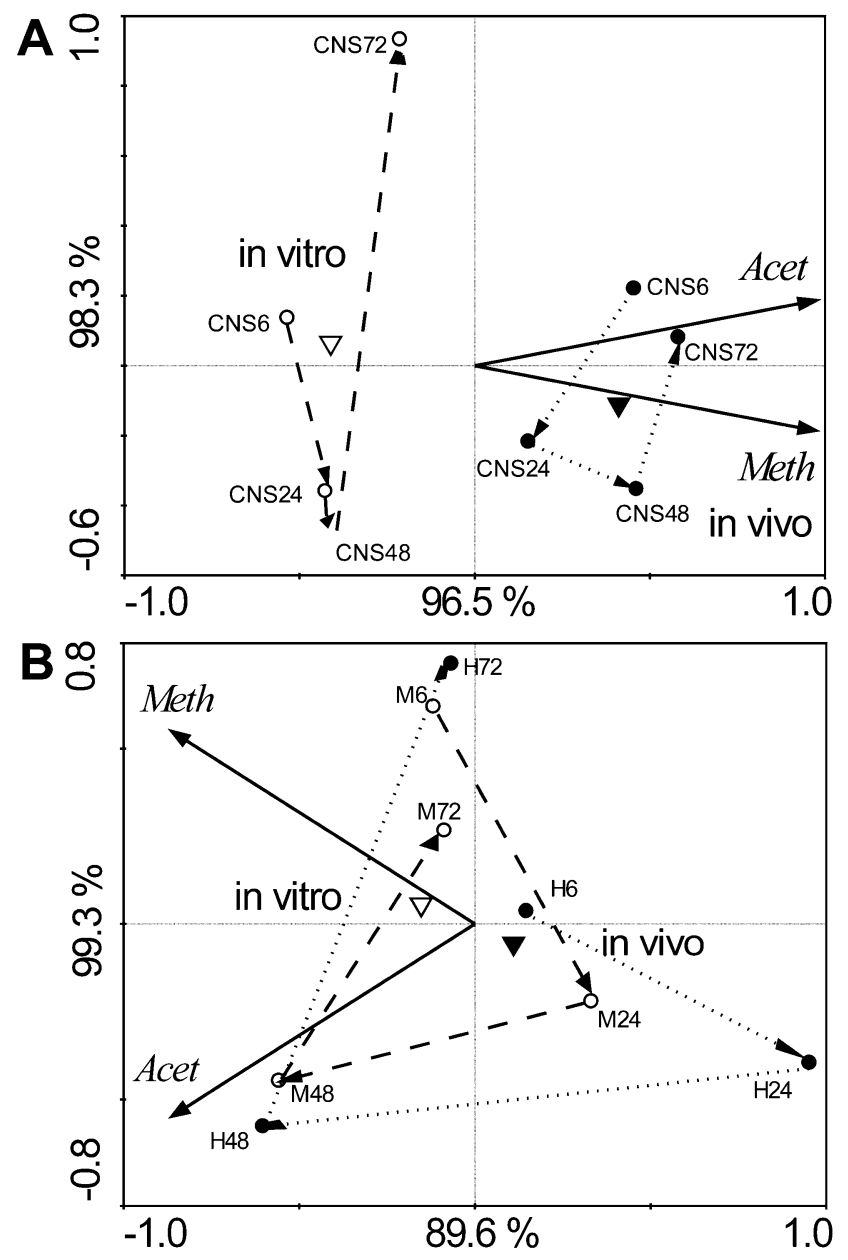

Fig. 4. The results of the multivariate principal component analysis of the differences between the in vivo and in vitro production of AKHs in CNS and their release into the haemolymph or medium after treatment with methoprene. A - change in the $\mathrm{AKH}$ content of the CNS, B - change in the AKH level in haemolymph (in vivo experiment) or medium (in vitro experiment). Abbreviations: CNS - (brain + CC + CA complex), $\mathrm{H}-$ haemolymph, $\mathrm{M}-$ medium, numbers behind $\mathrm{CNS}, \mathrm{H}$ and $\mathrm{M}$ are the times after the methoprene treatment, Acet - acetone, Meth - methoprene. Symbols: solid arrows - tendency of the AKH level to change after methoprene or acetone treatment, dotted arrows - tendency of the AKH level in the CNS (A) or in the haemolymph (B) in vivo to change after methoprene treatment, dashed arrows - tendency of the AKH level in the CNS (A) or medium (B) in vitro to change after methoprene treatment, triangles - represent central space projection of all data of the particular sets: solid triangles - in vivo sets, open triangles - in vitro sets. For details see text.

decrease in the release of AKH 24 (1.4 fold) and 48 (1.5 fold) $h$ after treatment was shown (Fig. 3B).

The question arises whether the production of AKHs in $\mathrm{CNS}$ and their release into the haemolymph under in vivo conditions and into the medium under in vitro conditions show the same characteristics. A multivariate principal component analysis (Fig. 4) corroborated the facts apparent from Figs 2 and 3 (increase in AKH content of CNS and decrease of AKH level in haemolymph or medium after treatment with methoprene), and revealed that the temporal pattern in the release of AKH from the CNS after treatment with methoprene, both in vivo and in vitro, is similar. Fig. 4A shows the distribution of AKH in the CNS after treatment with methoprene. The data below the central horizontal line show there were significant effects 24 and $48 \mathrm{~h}$ after the treatment both in vitro and in vivo. On the other hand the vectors and distances of the data (see dashed arrows vs. dotted arrows - Fig. 4A) display significant differences in their positions 24 and $48 \mathrm{~h}$ after the treatment, suggesting that another factor is involved. Whether other tissues or humoral factors in vivo have an effect remains speculative. Fig. 4B shows the results for haemolymph and medium, respectively: the trends in the changes mimic the situation in the CNS, with opposite directions of the methoprene effect; however, no significant difference between in vitro and in vivo mechanisms (as for CNS) was recorded.

\section{DISCUSSION}

Juvenile hormones are pleiotropic, controlling many aspects of insect life, but principally metamorphosis and, in many species, vitellogenesis and deposition of yolk in the oocytes (Nijhout, 1994). On the other hand, the adipokinetic peptides control predominantly energy metabolism but, being pleiotropic as well, also many other processes, including the synthesis of basic nutrients (inhibition of RNA, protein (vitellogenin) and lipid syntheses), interfering with actions not only of energy metabolism, but also with processes like oxidative stress, humoral and cellular immune response or reproduction (see Kodrík, 2008). It is therefore evident that functions of juvenile and adipokinetic hormones can intersect in some processes, and that a possible feedback between them is logical.

The results presented here indicate that treatment with methoprene resulted in a decrease in the level of AKH in the haemolymph and a corresponding increase in the CNS. These, at first appearance rather paradoxical results, suggest that the methoprene treatment may control AKH release, while the effect on AKH synthesis could be either small or, more likely, negligible. The higher AKH content in the CNS of methoprene-treated bugs could be a result of higher AKH accumulation. As described previously, there is a continuous spontaneous increase in the amounts of adipokinetic peptides in the CNS with age of $P$. apterus adults (Kodrík et al., 2003); however, the phenomenon is also known in other insect species. Studies on the total content of three different AKHs (Locmi-AKH-I, -II and -III) in the CC of nymphs and adults of L. migratoria showed a continuous increase in the amounts of these hormones (Bray et al., 1993; Oudejans et al., 1993), which is reflected in the increase in the number of secretory granules, at least in ageing adults (Diederen et al., 1992). The amounts of AKH-I and AKH-II continuously increase also in the CC during the life cycle of Schistocerca gregaria (Hekimi et al., 1991). Thus, it is likely that the level of AKH in the haemolymph fluctuates depending on the transitory demands of the organism and external conditions (locomotory activity, metabolic stress, 
photoperiodic rhythms, etc.), while the AKH level in the $\mathrm{CNS}$ is more stable, reflecting relatively slow (developmental and ageing) changes. The rapid changes in the level of $\mathrm{AKH}$ in the haemolymph also reflect the rapid turnover of AKHs. A half-life in the order of a few minutes is proposed for several insect species: for Pyrap-AKH in P. apterus 18 min (Goldsworthy et al., 2002) and Locmi-AKH-I, -II and -III in L. migratoria 51, 40 and $5 \mathrm{~min}$ at rest, and 35,37 and $3 \mathrm{~min}$ during flight (Oudejans et al., 1996; Van der Horst et al., 1999). All these data and the above conclusions accord with the suggestion that a coupling between release and biosynthesis of the AKHs in the adipokinetic cells is very loose or does not exist (Diederen et al., 2002).

The suppression of AKH release from the CNS into the haemolymph after treatment with methoprene, recorded in this paper accords with several findings on $P$. apterus adults of both sexes. There is a lower titre of AKH in the haemolymph of brachypterous reproductive females than in the macropterous morph (Kodrík et al., 2002b; Socha et al., 2005) during ovarian arrest (Socha \& Šula, 1996). When the CA is fully-active it is likely there is a higher level of JH-stimulated vitellogenin synthesis and ovarian growth (Socha et al., 1991). In adult males the same phenomenon is connected with the development of reproductive accessory glands in the brachypterous morph (Socha et al., 2004) and with delayed development of accessory glands in the macropterous morph (Socha, 2006) due to the inactivity of the CA (Hodková \& Socha, 2006; Socha \& Hodková, 2006).

Based on the level of lipids in the haemolymph it was concluded that methoprene treatment induced a significant reduction in the lipid content of the haemolymph of males ( $24 \mathrm{~h}$ after treatment). This accords with the observation that JH III and JHa suppress total level of 1,2-diacylglycerols in $S$. gregaria haemolymph (Schneider et al., 1995). The authors of this paper also showed, unlike our results for P. apterus, that $\mathrm{JH}$ and $\mathrm{JHa}$ suppress the adipokinetic response in the fat body of $S$. gregaria.

It is well documented that both $\mathrm{JH}$ and $\mathrm{AKH}$ control insect reproduction. JH stimulates (Engelmann, 1979; Nijhout, 1994) and AKH inhibits (Carlisle \& Loughton, 1986; Lorenz, 2003) vitellogenin synthesis and hence both hormones control ovarian maturation. Stimulation of vitellogenin synthesis by $\mathrm{JH}$ is a typical example of hormonally controlled gene expression that switches on complicated synthetic machinery in the fat body, which controls the transport of vitellogenin to the ovarioles and its entering into the maturing oocytes (Chapman, 1998). On the other hand, the AKHs selectively inhibit the level of vitellogenin synthesis (Moshitzky \& Applebaum, 1990) when the terminal oocytes are large. This effect is apparently independent of lipid mobilization, because inhibition occurs at hormone titres that are about one tenth of those required to initiate lipid mobilization, so the two actions of AKH are not coincident (Carlisle \& Loughton, 1986). Our preliminary experiments on the interactions between the functions of $\mathrm{JH}$ and $\mathrm{AKH}$ in females of the firebug $P$. apterus and the cotton bug $D$. cingulatus (Bártů et al., 2006) indicate an inhibiting effect of JHa on AKH characteristics, but the interpretation of the results are complicated by ovarian cycles and corresponding hormonal changes in females. Those problems were avoided by using males for the experiments, despite the fact that interesting phenomena, such as the interaction of the hormones with vitellogenin synthesis in females, could not be studied.

In summary, the present study showed that methoprene (1) inhibited the release of AKH from the CNS complex into the haemolymph, and (2) induced a partial reduction in the lipid content of the haemolymph in adult males of $P$. apterus. The increased levels of AKHs in the CNS complex are most likely the result of accumulation than stimulation of their synthesis.

ACKNOWLEDGEMENTS. This study was supported by grant No. 522/07/0788 from the Czech Science Foundation (DK), and the Institute of Entomology project No. Z50070508 funded by the Academy of Sciences of the Czech Republic. The authors thank D. Rienesslová and G. Štěpánová for their technical assistance, and A. Tomčala for the multivariate principal component analysis.

\section{REFERENCES}

BÁrtŮ I., PAtočKová M. \& Kodrík D. 2006: The effect of juvenile hormone analogue on adipokinetic hormone activity in bugs (Heteroptera, Insecta). (Published abstract) Physiol. Res. 55: 13-14.

Bray M.M., Shafi S., Wheeler C.H. \& Goldsworthy G.J. 1993: Quantification by radioimmunoassay of the adipokinetic hormone-I in neural tissues in the head of Locusta migratoria. Comp. Biochem. Physiol. (A) 106: 257-262.

Carlisle J. \& Loughton B.G. 1986: The inhibition of protein synthesis in Locusta migratoria by adipokinetic hormone. $J$. Insect Physiol. 32: 573-578.

Chapman R.F. 1998: The Insects. Structure and Function. 4th ed. Cambridge University Press, Cambridge, UK, 770 pp.

Diederen J.H.B., Peppelenbosch M.P. \& Vullings H.G.B. 1992: Ageing adipokinetic cells in Locusta migratoria: An ultrastructural morphometric study. Cell Tiss. Res. 268: 117-121.

Diederen J.H.B., Oudejans R.C.H.M., Harthoorn L.F. \& Van DER HORST D.J. 2002: Cell biology of the adipokinetic hormone-producing neurosecretory cells in the locust corpus cardiacum. Microsc. Res. Tech. 56: 227-236.

ENGELMANN F. 1979: Insect vitellogenin: identification, biosynthesis and role of vitellogenins. Adv. Insect Physiol. 14: 49-108.

Fernlund P. \& Josefsson L. 1972: Crustacean color-change hormone: Amino acid sequence and chemical synthesis. Science 177: $173-175$.

GÄDE G. \& Goldsworthy G.J. 2003: Insect peptide hormones: a selective review of their physiology and potential application for pest control. Pest Manag. Sci. 59: 1063-1075.

Gäde G., Hoffmann K.H. \& Spring J.H. 1997: Hormonal regulation in insects: facts, gaps, and future directions. Physiol. Rev. 77: 963-1032.

Goldsworthy G.J., Kodrík D., Comley R., Lightfoot M. 2002: A quantitative study of the adipokinetic hormone of the firebug, Pyrrhocoris apterus. J. Insect Physiol. 48: 1103-1109.

Hekimi S., Fischerlougheed J. \& Oshea M. 1991: Regulation of neuropeptide stoichiometry in neurosecretory cells. J. Neurosci. 11: 3246-3256. 
Hodková M. \& Socha R. 2006: Endocrine regulation of the reproductive arrest in the long-winged females of a flightless bug, Pyrrhocoris apterus (L.). Eur. J. Entomol. 103: 523-529.

HONĚK A. 1976: The regulation of wing polymorphism in natural populations of Pyrrhocoris apterus (Heteroptera, Pyrrhocoridae). Zool. Jb. Syst. 103: 547-570.

HONĚK A. 1985: Ecophysiological differences between brachypterous and macropterous morphs on Pyrrhocoris apterus (Heteroptera, Pyrrhocoridae). Acta Entomol. Bohemoslov. 82: 347-354.

KodRík D. 2008: Adipokinetic hormone functions that are not associated with insect flight. Physiol. Entomol. 33: 171-180.

Kodrík D., Socha R., Šimek P., Zemek R. \& Goldsworthy G.J. 2000: A new member of the AKH/RPCH family that stimulates locomotory activity in the firebug, Pyrrhocoris apterus (Heteroptera). Insect Biochem. Mol. Biol. 30: 489-498.

KodRík D., ŠIMEK P., LEPŠA L. \& SochA R. 2002a: Identification of the cockroach neuropeptide Pea-CAH-II as a second adipokinetic hormone in the firebug Pyrrhocoris apterus. Peptides 23: 583-585.

Kodrík D., Socha R. \& Syrová Z. 2002b: AKH content fluctuates in Pyrrhocoris apterus (L.) body. In Keller R., Dircksen H., Sedlmeier D. \& Vaundry H. (eds): Proceedings of the $21^{s t}$ Conference of European Comparative Endocrinologists. Monduzzi, Bonn, pp. 175-178.

Kodrík D., Socha R. \& Syrová Z. 2003: Developmental and diel changes of adipokinetic hormone in CNS and haemolymph of the flightless wing-polymorphic bug, Pyrrhocoris apterus (L.). J. Insect Physiol. 49: 53-61.

Kodrík D., Marco H.G., Šmek P., Socha R., Štys P. \& GÄdE G. 2010: The adipokinetic hormones of Heteroptera: A comparative study. Physiol. Entomol. 35: (DOI: 10.1111/j.1365-3032. 2009.00717.x).

LORENZ M.W. 2003: Adipokinetic hormone inhibits the formation of energy stores and egg production in the cricket Gryllus bimaculatus. Comp. Biochem. Physiol. (B) 136: 197-206.

Maxová A., Kodríx D., Zemek R. \& Socha R. 2001: Diel changes in adipokinetic response and walking activity of Pyrrhocoris apterus (L.) (Heteroptera) in relation to physiological status and wing dimorphism. Eur. J. Entomol. 98: 433-438.

Moshitzky P. \& Applebaum S.W. 1990: The role of adipokinetic hormone in the control of vitellogenins in locusts. Insect Biochem. 20: 319-323.

Nishout H.F. 1994: Insect Hormones. Princeton University Press, Princeton, NJ, 267 pp.

Oudejans R.C.H.M., Mes T.H.M., Koolman F.P. \& VAn DeR HoRst D.J. 1993: Adipokinetic peptide hormone content and biosynthesis during locust development. Peptides 14: 877-994.

Oudejans R.C.H.M., VRoemen S.F., Jansen R.F.R. \& Van DeR Horst D.J. 1996: Locust adipokinetic hormones: Carrierindependent transport and differential inactivation at physiological concentrations during rest and flight. Proc. Natl. Acad. Sci. USA 93: 8654-8659.

SchNeIDER M., Wisel G. \& Dorn A. 1995: Effects of JH III and $\mathrm{JH}$ analogue on phase-related growth, egg maturation and lipid metabolism in Schistocerca gregaria females. $J$. Insect Physiol. 41: 23-31.

Sláma K., RomanuK M. \& Šorm F. 1974: Insect Hormones and Bioanalogues, Springer, Wien, 477 pp.

Socha R. 1993: Pyrrhocoris apterus (Heteroptera) - an experimental model species: a review. Eur. J. Entomol. 90: 241-286.

SochA R. 2004: Decreased mating propensity of macropterous morph in a flightless wing-polymorphic insect, Pyrrhocoris apterus (L.) (Heteroptera). Eur. J. Entomol. 101: 539-545.
Socha R. 2006: Endocrine control of wing morph-related difference in mating success and accessory gland size in male firebugs. Anim. Behav. 71: 1273-1281.

SocHA R. 2007: Factors terminating ovarian arrest in longwinged females of a flightless bug, Pyrrhocoris apterus (Heteroptera: Pyrrhocoridae). Eur. J. Entomol. 104: 15-22.

SOCHA R. 2008: Wing morph- and age-related differences in fertilization success of adult males of a flightless bug, Pyrrhocoris apterus (Heteroptera: Pyrrhocoridae). Eur. J. Entomol. 105: 93-98.

Socha R. \& Hodková M. 2006: Corpus allatum volumedependent differences in accessory gland maturation in longand short-winged males of Pyrrhocoris apterus (Heteroptera: Pyrrhocoridae). Eur. J. Entomol. 103: 27-32.

Socha R. \& Šula J. 1996: Differences in haemolymph proteins in relation to diapause and wing dimorphism in Pyrrhocoris apterus (L.) (Heteroptera: Pyrrhocoridae). J. Comp. Physiol. (B) 166: 382-387.

Socha R. \& Šula J. 2006: Flight muscles polymorphism in a flightless bug, Pyrrhocoris apterus (L.): Developmental pattern, biochemical profile and endocrine control. J. Insect Physiol. 52: 231-239.

Socha R. \& Šula J. 2008: Regulation of the development of flight muscles in long-winged adults of the flightless bug, Pyrrhocoris apterus (Heteroptera: Pyrrhocoridae). Eur. J. Entomol. 105: 575-583.

Socha R. \& ZeMEK R. 2000: Locomotor activity in adult Pyrrhocoris apterus (Heteroptera) in relation to sex, physiological status and wing dimorphism. Physiol. Entomol. 25: 383-389.

Socha R. \& Zемек R. 2003: Wing morph-related differences in the walking pattern and dispersal in a flightless bug, Pyrrhocoris apterus (L.) (Heteroptera). Oikos 100: 35-43.

Socha R. \& ZemeK R. 2007: Temporal pattern of feeding activity in the firebug Pyrrhocoris apterus and its relation to sex, wing dimorphism and physiological state of adults. Physiol. Entomol. 32: 16-25.

Socha R., Šula J. Kodrík D. \& Gelbič I. 1991: Hormonal control of vitellogenin synthesis in Pyrrhocoris apterus (L.) (Heteroptera). J. Insect Physiol. 37: 805-816.

Socha R., Śula J. \& ZEMEK R. 1997: Feeding, drinking and digestive enzyme activities in short- and long-day females of Pyrrhocoris apterus (Heteroptera). Physiol. Entomol. 22: 161-169.

Socha R., Ś Ula J. \& ZemeK R. 1998: Feeding behaviour, digestive physiology and lipid content in macropterous females of Pyrrhocoris apterus (L.) (Heteroptera: Pyrrhocoridae). Physiol. Entomol. 23: 91-96.

Socha R., Šula J. \& Kodrík D. 2004: Wing morph-related differences in developmental pattern of accessory gland proteins in adult males of Pyrrhocoris apterus (L.) and their endocrine control. J. Insect Physiol. 50: 893-901.

Socha R., Kodrík D. \& Šula J. 2005: Wing morph-specific differences in the metabolism and endocrine control of reserve mobilisation in adult males of a flightless bug, Pyrrhocoris apterus (L.) (Heteroptera). J. Comp. Physiol. (B) 175: 557-565.

Van der Horst D.J., Van MarrewiJK W.J.A., Vullings H.G.B. \& Diederen J.H.B. 1999: Metabolic neurohormones: Release, signal transduction and physiological responses of adipokinetic hormones in insects. Eur. J. Entomol. 96: 299-308.

ZÖLLNER N. \& KIRSCH K. 1962: Über die quantitative Bestimmung von Lipoide (Mikromethode) mittels der vielen natürlichen Lipoiden (allen bekannten Plasmalipoiden) gemeinsamen SulfophosphovanillinReaktion. Z. Ges. Exp. Med. 135: $545-561$.

Received July 22, 2009; revised and accepted September 30, 2009 